\title{
Diet and cognition: Data, theory, and some solutions from the playbook of psychology.
}

Consumption of foods made from highly processed and refined ingredients has been identified as one of the leading causes of poor physical health and disease, such as obesity, diabetes, metabolic syndrome, cancer, degenerative disease, cardiovascular disease, and autoimmune disease [1]. In addition to its effects on physical health, I describe here how diet also plays a major role in mood, cognition, and psychological wellbeing. In my talk, I first address the question: How does eating refined food cause cognitive impairments and contribute to mood disorders? Next, I address the question of why individuals continue to consume refined food given its negative impact on physical and mental health. I close with a discussion of how we can use psychological concepts as tools to help remove refined foods from the diet.

\section{How does eating refined food cause cognitive impairments?}

Rodent studies show that consuming a refined-food (REF) diet leads to many cognitive impairments. In work from my lab, we've compared the effects in rats of consuming a low-fat REF diet to a standard, unrefined low-fat control (CON) diet on physical health and cognition. The fats, carbohydrates, and proteins that constitute the CON diet are derived from whole-food sources while those of the REF diet are derived from isolated, refined, and purified sources. Rats that consume a REF diet over many months gain a high degree of adiposity. Furthermore, consumption of the REF diet causes impairments in motivation [2], and in sustained attention and impulse control [3]. Commonly-used REF rodent diets that are high in both sugar and fat (the so-called 'Western diet') also produce deficits in working memory [4]; and hippocampal-dependent learning tasks, such as configural learning [5] and spatial cognition [6]. REF diets have been shown to cause increased permeability of the blood brain barrier (BBB) near the hippocampus, but not near other higher brain areas, such as striatum and frontal cortex. This selective BBB permeability near the hippocampus provides a plausible causal mechanism for the many diet-induced cognitive deficits on hippocampal tasks [5].

\section{How does eating refined food contribute to mood disorders?}

Consumption of REF diets has also been associated with mood disorders. The mechanistic basis for this association may lie in part on the fact that refined ingredients, such as sugar and flour [7] and seed oils [8], have also been shown to increase systemic inflammation by stimulating cytokine production [9]. In turn, chronically-elevated inflammatory signaling may result in dysregulation of the production and metabolism of neurotransmitters and neuromodulators involved in the regulation of mood, such as dopamine, glutamate, and serotonin. Furthermore, inflammation-mediated depression resembles "sickness" behavior, such as social withdrawal and energy conservation [9], rather than a clinical depression which instead is characterized by anhedonia as a primary feature. Several micronutrient deficiencies are associated with anxiety and depression, such as zinc, magnesium, lithium, and chromium [10]. Refined foods are typically deficient in many micronutrients. On the other hand, consuming nutrient dense foods is associated with positive affect [11].

\section{Why do we eat refined foods?}


There are many contributing factors that promote selection of REF food over whole foods. The following list identifies some of the major psychological factors.

1. Refined foods are supernormal stimuli (hyperpalatable). According to ethologists, an artificial stimulus that elicits a greater response than a normal stimulus is called a supernormal stimulus [12]. For example, an oystercatcher is a ground nesting bird that tends to her eggs. If an artificial egg that is larger and more speckled than one of her own eggs is placed next to her nest, she will prefer to tend the artificial egg over her own real eggs [12]. Refined foods are hyperpalatable and elicit a consummatory response to a greater extent than natural, whole foods, thereby dysregulating appetite.

2. Pavlovian flavor-calorie associations (cravings). We learn what foods to eat by associating their flavors and odors with the calories they contain, that is, Pavlovian food preferences [13]. Refined foods are calorically dense, and thus establish much stronger flavor-calorie associations. As a result, we like these flavors much more and develop cravings for them [14].

3. More rapid habit learning (addiction). As we learn a new skill, we start by intently focusing on the new movements. With practice, the behavior turns into a habit. Diet can affect the speed at which this transition occurs. Rats consuming a REF diet showed faster habit learning than rats eating a CON diet [15]. In humans, obesity is associated with having more neurons in brain regions that govern habit learning [16], as well as quicker [16] and stronger [17] acquisition of new habits. Eating refined foods dysregulates appetite and food seeking behavior [18][19].

4. Impaired delay discounting (eroded willpower). We are usually able to forgo immediate rewards if we value a future outcome even more, such as avoiding a piece of cake now to attain a longer term weight loss goal. But we tend to discount rewards that are too far in the future, or when the immediate reward is too enticing, a phenomenon that is referred to as "Delay discounting". Obese individuals are at increased risk of losing control over their actions when those actions earn immediate incentives such as food or money, and the rate at which outcomes are discounted over time is more rapid in obese than lean individuals [20].

5. Learned helplessness (yo-yo dieting). Prior exposure to inescapable shock impairs shock avoidance learning in animals [21]. This phenomenon of learned helplessness may aptly account for the experience many people have with yo-yo dieting, by which forced calorie restriction achieves short-term but not sustainable weight loss [22]. Repeated unsuccessful attempts to lose weight through dieting can result in feelings of helplessness about maintaining a desired weight. As a result, an individual may give up trying to lose weight. Unlike Western diets or other REF diets, low-carbohydrate and whole-foods diets have been found to produce long-term sustainable weight loss by normalizing hunger and appetite [23].

6. Overshadowing impairs liking of whole foods. Pavlov studied how dogs learn to associate a cue, such as a bell, with an important outcome, like food. When there are multiple cues present, however, the dogs only learned to associate the most salient one with food. The less salient cues were overshadowed [24]. The strong flavors in REF foods may overshadow the milder flavors of whole foods, impairing the development of Pavlovian preferences for whole foods. 
7. Counterconditioning of bad tastes. Counterconditioning occurs when a cue that used to signal something bad is conditioned to signal something good. Many chemical additives in refined foods taste bad on their own, but when combined with lots of sugar, they can start to taste good through flavorcalorie learning.

8. Sensory adaptation and habituation. Overstimulation can lead to either sensory adaptation or habituation. Sensory adaptation involves the downregulation of sensory receptors as a result of intense stimulation. Consuming supernormal foods overstimulates taste receptors, leading to sensory adaptation [23]. As a result, natural flavors don't taste as good and we fail to find pleasure in eating whole foods.

Habituation is a learning process by which we come to ignore a persistent stimulus. We habituate to the fatigue, aches, and pains that are typical of aging, and thus fail to notice these signals of poor health.

\section{How can we banish refined foods from our diet?}

Despite these factors that contribute to unhealthy eating, psychology also provides the tools to gain control over our dietary choices to promote healthy eating behaviors.

1. Extinction of food cue associations. Pavlov showed that dogs learned to salivate to a bell that predicted food. Presenting the bell by itself repeatedly, however, would extinguish this conditioned response [24]. Presenting a food cue but without eating can break the prior association of that cue with the food. Food cues can include visual, auditory (rustling of food packaging), and odors and taste cues.

2. Break food cravings. Seth Roberts discovered that eating flavorless calories breaks flavor-calorie associations, thereby reducing appetite (by lowering the body fat set point) [25]. One effective method involves consuming 1 to 2 tablespoons of refined coconut oil daily in the absence of any flavors for at least an hour before or after. This method has yielded many cases of successful, long-term, and sustainable weight loss (see http://sethroberts.net/).

3. Reward devaluation (turn good into bad). A rat learns to press a lever for a sugar pellet. If the sugar pellet is followed by a stomach ache the rat will stop pressing the lever [26]. Devaluing REF foods can reduce our consumption of them, for example by thinking about the food one wants to devalue as being bad or toxic can be effective (see the next point).

4. Equivalence class formation (reclassify refined foods as toxins). We categorize perceptually dissimilar objects, such as tools, into equivalence categories based on a common function [27]. Members of one equivalence class can be reassigned to another. Thus, we can reclassify REF foods, such as donuts from the "food" category to the "toxic" category, and thereby change the donut from desirable to dislike. Likewise, organ meats can be reclassified as nutritious food to facilitate their inclusion in the diet.

5. New habit learning to replace unwanted habits. Consistent training establishes a habit. Habits are easy to follow because they don't require will power. Make a habit of avoiding REF foods and choosing whole foods. This overlooked strategy can be one of the most effective psychological hacks. 
6. Mindfulness and meditation. Mindfulness is a powerful shaper of individual behavior [28]. Be mindful of what, where, when, and how you choose to eat. See Dr. Brian Wansink's excellent website for mindfulness practices for healthy eating http://mindlesseating.org/what can i do.php.

7. Social support and networks. You're not in this alone! Surround yourself with supportive friends and family. Seek out a community that supports your goals.

8. Redefine yourself (identity makeover). This may sound smarmy, but if you don't like how you are, change who you are. You are NOT that chubby boy, fat girl, poster child for junk food, or perpetually fatigued professor! Instead, view yourself as a vibrant, healthy person no longer controlled by food. You are the one at the party who NEVER touches the cookies or drinks the soda. Self-revaluation is a potent force to effect positive change in your life. Do not underestimate the power of the mind, including the use of ideation, in effecting positive change.

In closing, in a recent study from my lab, rats that had become obese after consuming a REF diet for 9 months were switched to a whole-foods CON diet for 5 weeks, and they regained their motivation to work for rewards. Thus, it is possible to reverse some of the cognitive impairments induced by REF food by switching to a whole-foods diet.

\section{References}

[1] S. Lindeberg, Food and western disease: Health and nutrition from an evolutionary perspective, Wiley-Blackwell, Oxford, 2010.

[2] A.P. Blaisdell, Y. Lam, M. Lau, E. Telminova, H. Cheei, B. Fan, et al., Physiology \& Behavior Food quality and motivation : A re fi ned low-fat diet induces obesity and impairs performance on a progressive ratio schedule of instrumental lever pressing in rats, Physiol. Behav. 128 (2014) 220225. doi:10.1016/j.physbeh.2014.02.025.

[3] A.P. Blaisdell, T. Biedermann, E. Sosa, A. Abuchaei, N. Youssef, S. Bradesi, An obesogenic refined low-fat diet disrupts attentional and behavioral control processes in a vigilance task in rats, 2016.

[4] C.E. Greenwood, G.W. A, Learning and Memory Impairment in Rats Fed a High Saturated Fat Diet, 87 (1990) 74-87.

[5] S.E. Kanoski, Y. Zhang, W. Zheng, T.L. Davidson, The effects of a high-energy diet on hippocampal function and blood-brain barrier integrity in the rat, J. Alzheimer's Dis. 21 (2010) 207-219.

[6] D.M.D. Tran, R.F. Westbrook, Rats Fed a Diet Rich in Fats and Sugars Are Impaired in the Use of Spatial Geometry, Psychol. Sci. . 26 (2015) 1947-1957. doi:10.1177/0956797615608240.

[7] I. Spreadbury, Comparison with ancestral diets suggests dense acellular carbohydrates promote an inflammatory microbiota, and may be the primary dietary cause of leptin resistance and obesity, Diabetes, Metab. Syndr. Obes. Targets Ther. 5 (2012) 174-189.

[8] A.P. Simopoulos, Evolutionary aspects of Diet: The omega-6/omega-3 ratio and the brain, Mol. Neurobiol. 44 (2011) 203-215. doi:10.1007/s12035-010-8162-0.

[9] J.K. Kiecolt-Glaser, H.M. Derry, C.P. Fagundes, Inflammation: Depression fans the flames and 
feasts on the heat, Am. J. Psychiatry. 172 (2015) 1075-1091.

doi:10.1176/appi.ajp.2015.15020152.

[10] K. Młyniec, C.L. Davies, I.G. de Agüero Sánchez, K. Pytka, B. Budziszewska, G. Nowak, Essential elements in depression and anxiety. Part I., Pharmacol. Rep. 66 (2014) 534-44.

doi:10.1016/j.pharep.2014.03.001.

[11] A. Perez-Cornago, M.A. Zulet, J.A. Martinez, Association between mood and diet quality in subjects with metabolic syndrome participating in a behavioural weight-loss programme: A crosssectional assessment., Nutr. Neurosci. 18 (2014) 137-145.

doi:10.1179/1476830514Y.0000000116.

[12] N.. Tinbergen, Study of Instinct, 1951.

[13] P. Rozin, D. Zellner, The Role of Pavlovian Conditioning in the Acquisition of Food Likes and Dislikes, Ann. N. Y. Acad. Sci. 443 (1985) 189-202.

[14] S. Roberts, What makes food fattening? A Pavlovian theory of weight control, 2005.

[15] T.M. Furlong, H.K. Jayaweera, B.W. Balleine, L.H. Corbit, Binge-Like Consumption of a Palatable Food Accelerates Habitual Control of Behavior and Is Dependent on Activation of the Dorsolateral Striatum, J. Neurosci. 34 (2014) 5012-5022. doi:10.1523/JNEUROSCI.3707-13.2014.

[16] A. Horstmann, F.P. Busse, D. Mathar, K. Müller, J. Lepsien, H. Schlögl, et al., Obesity-Related Differences between Women and Men in Brain Structure and Goal-Directed Behavior., Front. Hum. Neurosci. 5 (2011) 58. doi:10.3389/fnhum.2011.00058.

[17] A. Horstmann, A. Dietrich, D. Mathar, M. Pössel, A. Villringer, J. Neumann, Slave to habit? Obesity is associated with decreased behavioural sensitivity to reward devaluation, Appetite. 87 (2015) 175-183. doi:10.1016/j.appet.2014.12.212.

[18] S.H. Ahmed, N.M. Avena, K.C. Berridge, A.N. Gearhardt, K. Guillem, Food Addiction, (2013) 28332858. doi:10.1007/978-1-4614-1997-6.

[19] K.C. Berridge, "Liking" and "wanting" food rewards: Brain substrates and roles in eating disorders, Physiol. Behav. 97 (2009) 537-550. doi:10.1016/j.physbeh.2009.02.044.

[20] R.E. Weller, E.W. Cook, K.B. Avsar, J.E. Cox, Obese women show greater delay discounting than healthy-weight women, Appetite. 51 (2008) 563-569. doi:10.1016/j.appet.2008.04.010.

[21] M.E. Seligman, Learned helplessness, Annu. Rev. Med. (1972) 207-412. doi:10.1146/annurev.me.23.020172.002203.

[22] T. Mann, A.J. Tomiyama, E. Westling, A. Lew, B. Samuels, J. Chatman, Medicare's Search for Effective Obesity Treatments, 62 (2007) 220-233. doi:10.1037/0003-066X.62.3.220.

[23] E.C. Westman, R.D. Feinman, J.C. Mavropoulos, M.C. Vernon, J.S. Volek, J.A. Wortman, et al., Low-carbohydrate nutrition and metabolism, Am. J. Clin. Nutr. 86 (2007) 276-284. doi:86/2/276 [pii].

[24] I.P. Pavlov, Conditioned Reflexes, 1927. doi:10.2307/1134737. 
[25] S. Roberts, The Shangri-La diet: The no hunger eat anything weight-loss plan, Penguin, 2007.

[26] J. Garcia, D.J. Kimeldorf, R. a Koelling, Conditioned aversion to saccharin resulting from exposure to gamma radiation., Science. 122 (1955) 157-158. doi:10.1126/science.122.3179.1089.

[27] P.J. Urcuioli, Associative symetry, antisymetry, and a theory of pigeons' equivalence class formation, J. Exp. Anal. Behav. 90 (2008) 257-282. doi:10.1901/jeab.2008.90-257.

[28] K.W. Brown, R.M. Ryan, J.D. Creswell, Mindfulness: Theoretical Foundations and Evidence for its Salutary Effects, Psychol. Inq. 18 (2007) 211-237. doi:10.1080/10478400701598298. 